\title{
Effects of Consumption of Various Fatty Acids on Serum HDL-Cholesterol Levels
}

\author{
Hidekatsu Yanai ${ }^{\mathrm{a}, \mathrm{c}}$, Norio Tada ${ }^{\mathrm{b}}$
}

\begin{abstract}
Since HDL is an anti-atherogenic lipoprotein which plays a role in reversing cholesterol transport from the peripheral tissues to the liver, low HDL-cholesterol (HDL-C) levels are associated with the development of cardiovascular diseases. We previously studied effects of intake of various dietary fat on serum HDL-C levels to make "Dietary Reference Intakes for Japanese 2015", and found that the substitution of fatty acids (FAs) for carbohydrates is beneficially associated with HDL-C, monounsaturated FA (MUFA) intake may not affect HDL-C and trans FA (TFA) is significantly associated with reduction of HDLC. Consumption of n-3 poly-unsaturated FA (PUFA) was favorably associated with HDL-C. Here we review meta-analyses on the effects of various FA consumption on serum HDL-C levels, to make "Dietary Reference Intakes for Japanese 2020". Consumption of ruminantTFA may not affect HDL; however, increased industrially produced TFA intake was associated with a significant decrease in HDL and a significant increase in LDL. An intake of n-3 PUFA and MUFA was associated with an increase of HDL. An intake of saturated FA (SFA) was associated with an increase of HDL; however, SFA was also associated with an increase of LDL.
\end{abstract}

Keywords: High-density lipoprotein; Monounsaturated fatty acids, Poly-unsaturated fatty acids; Saturated fatty acids; Trans fatty acids

\section{Introduction}

Atherogenic dyslipidemia is characterized as elevated serum levels of triglyceride (TG) and low-density lipoprotein-cholesterol (LDL-C), and low serum levels of high-density lipoprotein-cholesterol (HDL-C). Since HDL is an anti-atherogenic lipoprotein which plays a role in reversing cholesterol transport from the peripheral tissues to the liver, low HDL-C levels

Manuscript submitted September 11, 2018, accepted September 21, 2018

aDepartment of Internal Medicine, National Center for Global Health and Medicine Kohnodai Hospital, Chiba, Japan

${ }^{\mathrm{b}}$ The Jikei University School of Medicine, Tokyo, Japan

${ }^{\mathrm{c} C}$ Corresponding Author: Hidekatsu Yanai, Department of Internal Medicine, National Center for Global Health and Medicine Kohnodai Hospital, 1-7-1 Kohnodai, Chiba 272-8516, Japan. Email: dyanai@hospk.ncgm.go.jp

doi: https://doi.org/10.14740/jem534 are associated with the development of coronary heart diseases (CHDs) [1, 2], and all-cause mortality [3].

We previously studied effects of intake of various dietary fat on serum HDL-C levels to make "Dietary Reference Intakes for Japanese 2015", by using meta-analyses of clinical trials which evaluated effects of various dietary fat consumption on HDL-C [4]. We found that the substitution of fatty acids (FAs) for carbohydrates is beneficially associated with HDL metabolism, monounsaturated FA (MUFA) intake may not affect HDL-C and trans FA (TFA) is significantly associated with reduction of HDL-C. Consumption of n-3 polyunsaturated fatty acids (PUFA), especially docosahexaenoic acid (DHA) consumption, was favorably associated with HDL metabolism.

Here we review meta-analyses on the effects of various FA consumption on serum HDL-C levels, to make "Dietary Reference Intakes for Japanese 2020".

\section{Materials and Methods}

To make "Dietary Reference Intakes for Japanese 2020", we searched meta-analyses of randomized controlled trial (RCT). A search was conducted by using PubMed, Embase and Google Scholar, with the following keywords: trans FA (TFA) and HDL and meta-analysis or saturated FA (SFA) and HDL and meta-analysis or MUFA and HDL and meta-analysis. The search period was comprised from 2012 up to July 2018.

\section{Results}

Meta-analyses which evaluated effects of various FA consumption on HDL-C were shown in Table 1.

\section{Palm oil, SFA}

Compared with most other vegetable oils such as olive and sunflower oils, palm oil contains a high amount of SFA (40$50 \%$ of total fat) with the majority being in the form of palmitic acid (16:0). Palm oil consumption significantly increased LDL-C compared with vegetable oils low in SFA [5]. Further, palm oil increased HDL-C by $0.02 \mathrm{mmol} / \mathrm{L}$ compared with vegetable oils low in SFA and by $0.09 \mathrm{mmol} / \mathrm{L}$ compared with TFA-containing oils. 

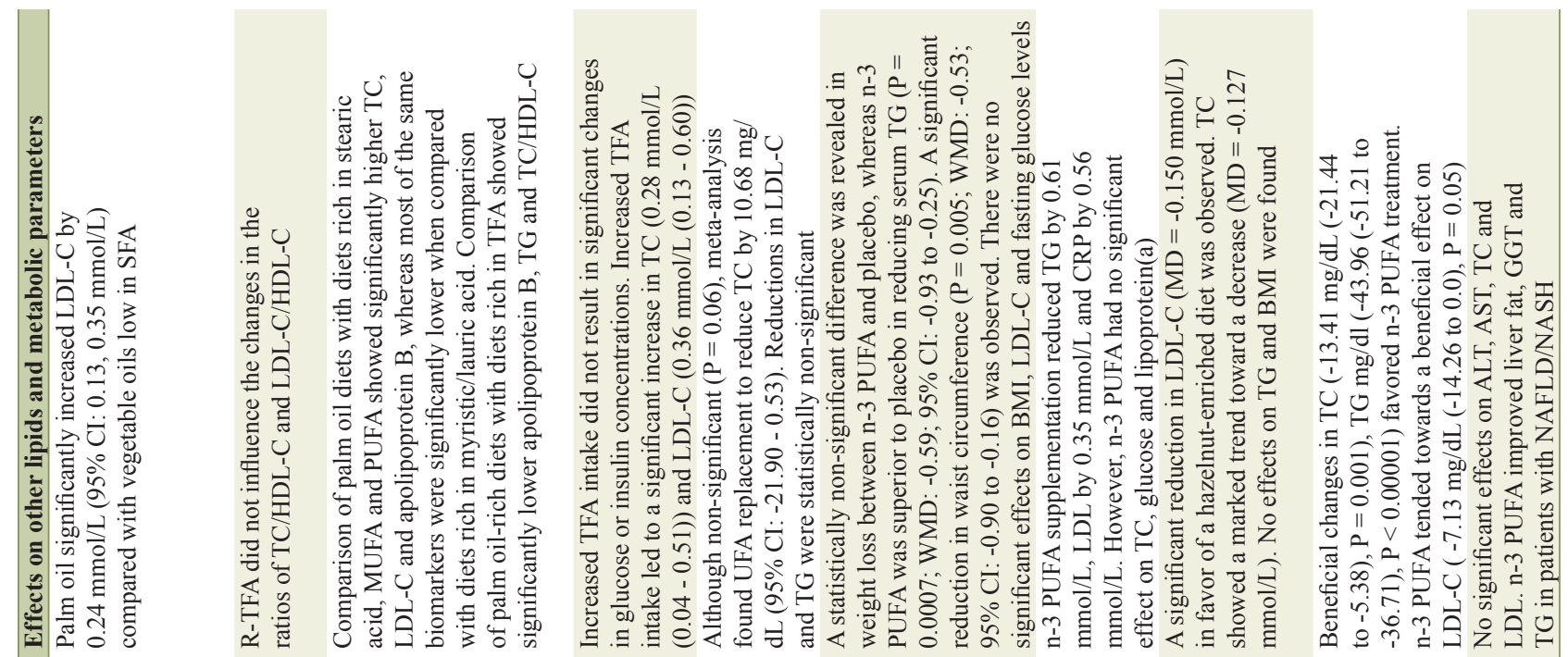

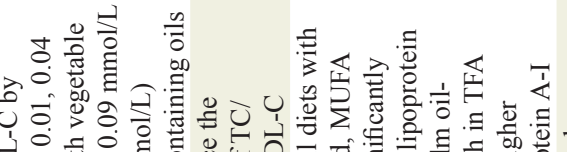

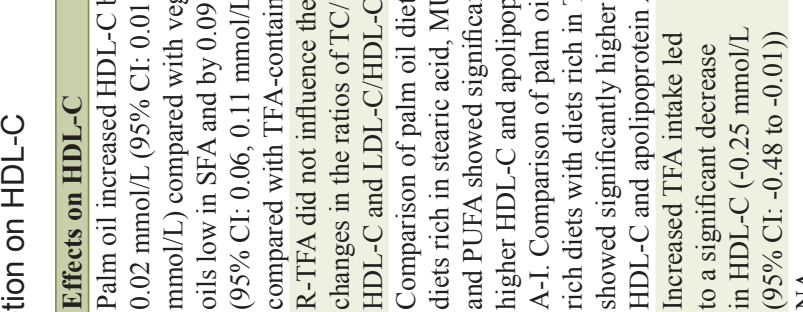
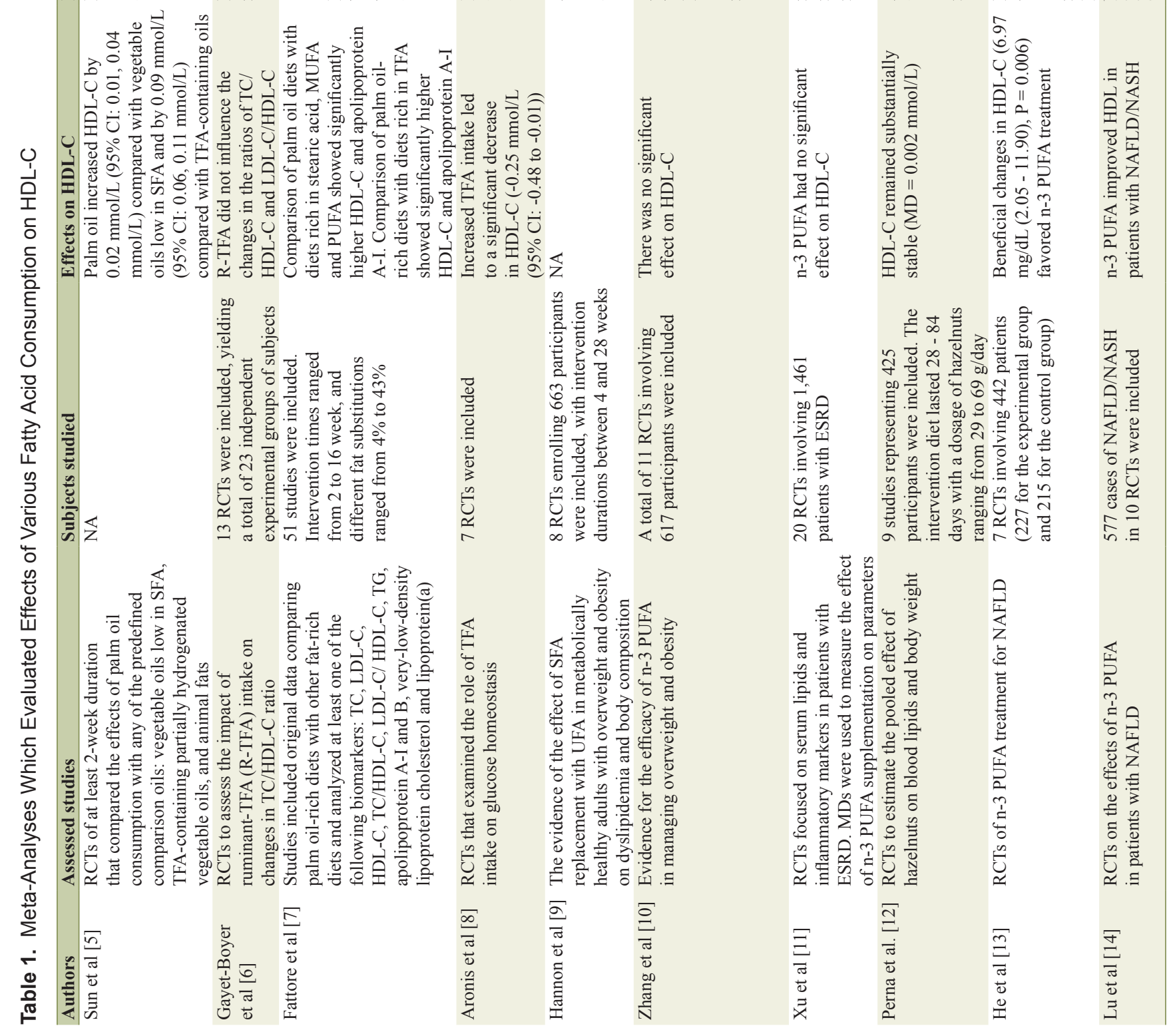

ลิ) 


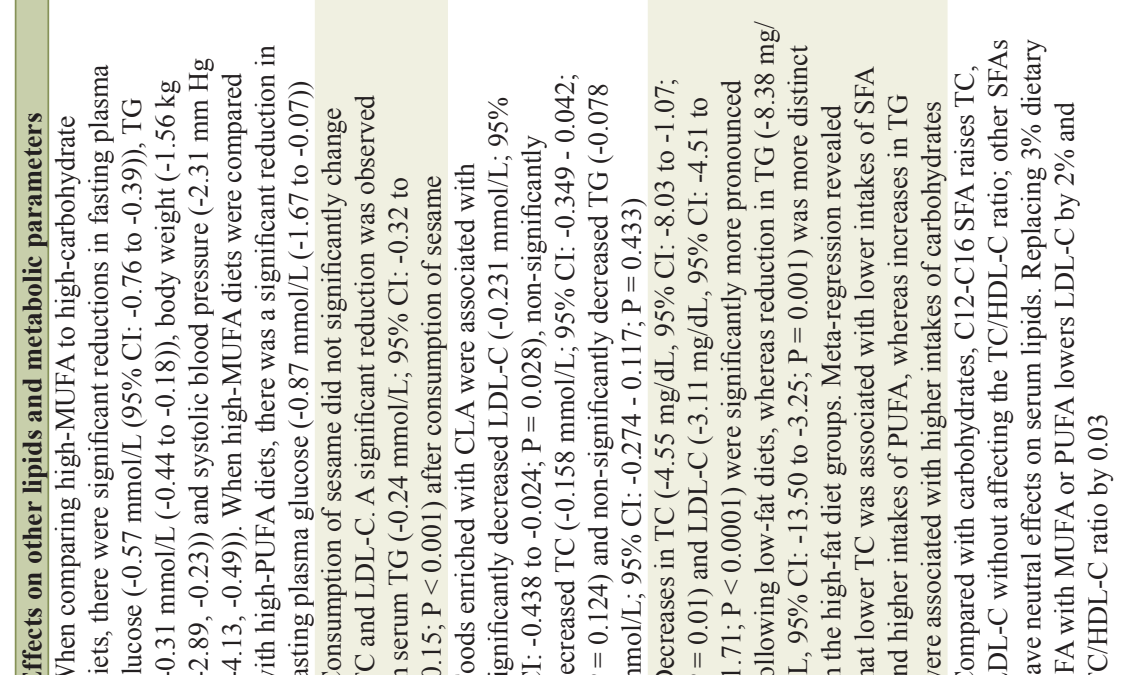

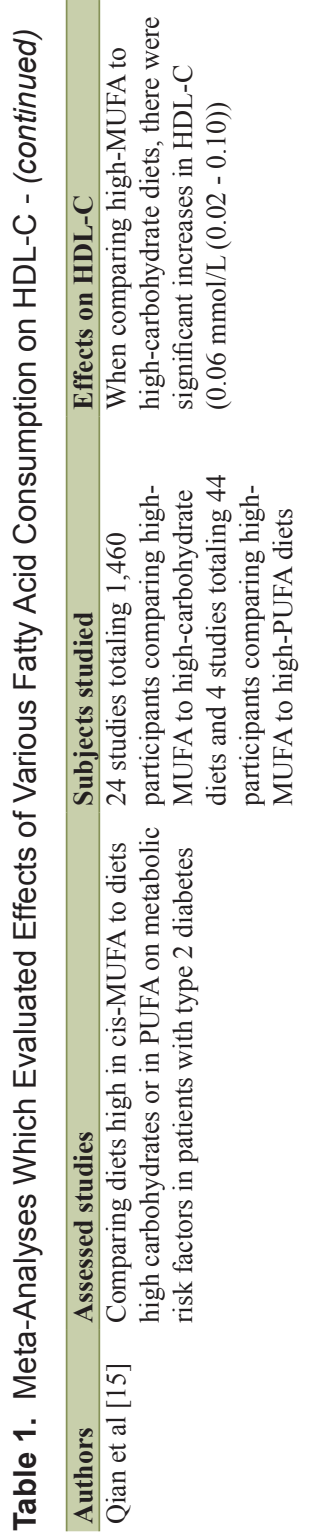

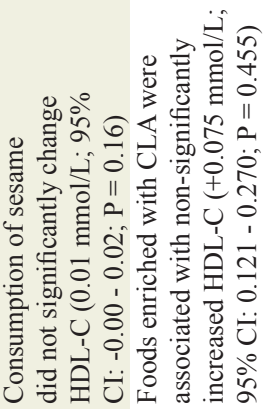

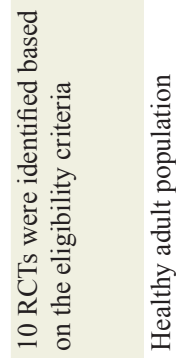

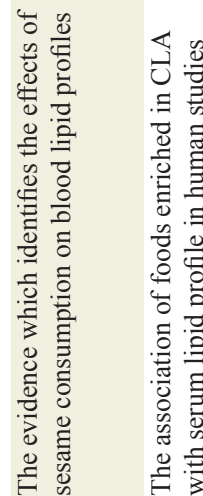

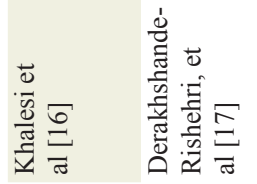

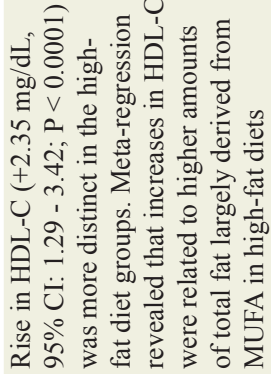

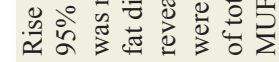

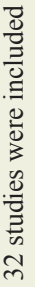

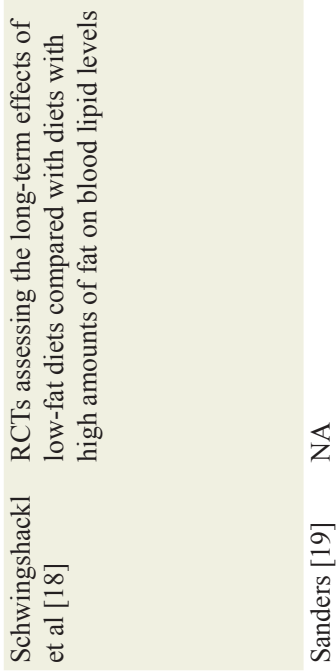

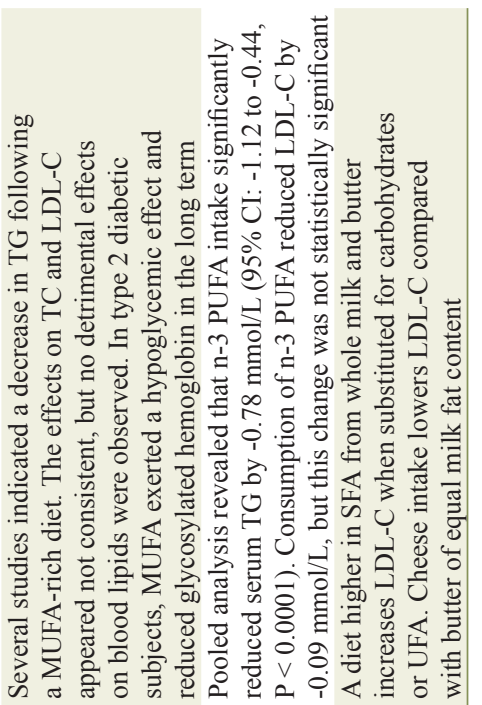

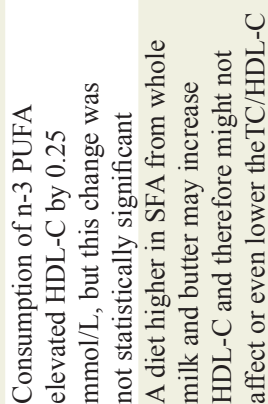

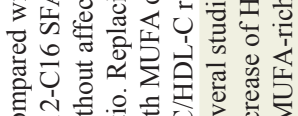

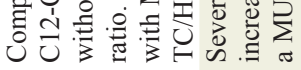
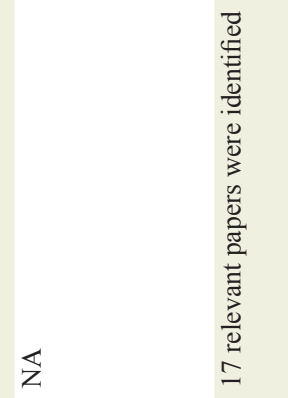

in

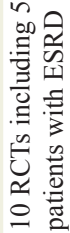

乙
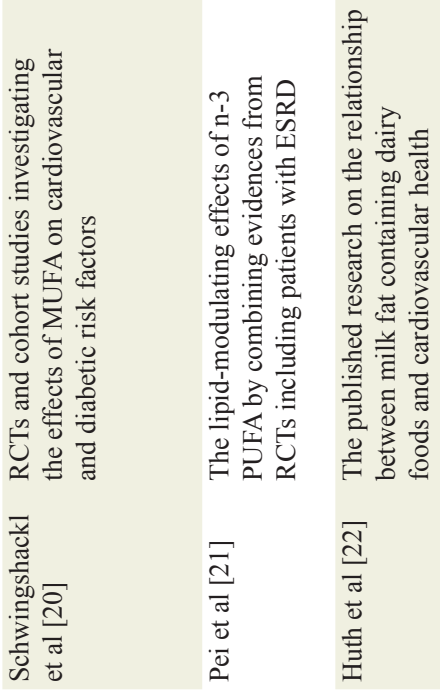

高䆓

1 in

$\rightarrow \frac{\pi}{2} \pi$

잉 응 要

of $\frac{0}{0}$

क क

0.0

这

응

음ㅎㅁ

辛势

क्षे

놀

ن⿺辶一

오휴휴

बi

क्ष $\frac{0}{\overline{0}} \frac{\infty}{\pi}$

要

入

密

क्षे

零 $\frac{0}{0}$

है응

$\therefore \frac{1}{\circ} \frac{\pi}{0}$

0

(1)

号芒齐

需

焉恋语

它

它莡岳

员兽离

山⿸丆口广

응 흐을

잉

일

क षे है

त.

可

家家

过市

更

更 $\sum$

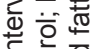

要

更

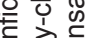

记 
In another meta-analysis of palm oil on serum lipids, comparison of palm oil diets with diets rich in stearic acid which is a long chain (C18:0) dietary SFA, MUFA and PUFA showed significantly higher HDL-C and apolipoprotein A-I [7]. Comparison of palm oil-rich diets with diets rich in TFA showed significantly higher concentrations of HDL-C and apolipoprotein A-I.

Compared with carbohydrates, C12-C16 SFAs raise serum LDL-C and HDL-C without affecting the TC/HDL-C ratio; other SFAs have neutral effects on serum lipid profile [19]. Results from short-term intervention studies on serum lipids have indicated that a diet higher in SFA from whole milk and butter increases LDL-C when substituted for carbohydrates or unsaturated FA (UFA); however, they may also increase HDL$\mathrm{C}$ and therefore might not affect or even lower the TC/HDL-C ratio [22].

\section{TFA}

Since the 1990s, TFAs have been reported to link to harmful effects, as they induce not only an increase in LDL-C but also a decrease in HDL-C [23]. The generic term of TFA represents two independent dietary sources, i.e. an industrial one (industrially produced TFA; IP-TFA) and a natural counterpart (ruminant TFA; R-TFA). R-TFAs are generally present in food at low levels (up to $8 \%$ of total FA in milk fat), whereas IP-TFA may reach up to $61 \%$ of total FA in pastries and shortenings [24]. R-TFA includes vaccenic acid and conjugated linoleic acid (CLA) that are synthesized by rumen bacteria via the metabolism of MUFA and PUFA [25-27].

The meta-analysis of R-TFA on serum lipids showed that doses of R-TFA did not influence the changes in the ratios of plasma TC/HDL-C and LDL-C/HDL-C [6]. Foods enriched with CLA were associated with a non-significant increase of HDL-C [17]. The meta-analysis showed that increased TFA (IP-TFA) intake led to a significant increase in LDL-C and a significant decrease in HDL-C [8].

\section{n-3 PUFA}

In the meta-analysis to evaluate evidence for the efficacy of n-3 PUFA in managing overweight and obesity, a significant reduction in waist circumference was obtained; however, a significant effect of n-3 PUFA on HDL-C was not observed [10]. The meta-analysis of n-3 PUFA on serum lipids in patients with end-stage renal disease (ESRD) also failed to prove a statistically significant beneficial effect of n-3 PUFA on HDL [11, 21]. However, two meta-analyses to identify the effectiveness of n-3 PUFA in non-alcoholic fatty liver disease (NAFLD) showed that n-3 PUFA improved HDL metabolism [13, 14].

\section{Hazelnuts, sesame, MUFA}

Hazelnuts are rich in MUFA and antioxidant bioactive substance. The meta-analysis of effect of hazelnuts on blood lipids did not show a significant effect of hazelnuts on HDL [12].

Sesame contains considerable amounts of vitamin E, MUFA, dietary fiber and lignans, which are thought to be associated with its plasma lipid-lowering properties. Consumption of sesame did not significantly change HDL-C [16].

When comparing high-MUFA to high-carbohydrate diets, there were significant increases in HDL-C [15]. Rise in HDL-C was more distinct in the high-fat diet groups, and meta-regression revealed that increases in HDL-C were related to higher amounts of total fat largely derived from MUFA in high-fat diets [18].

According to the synopsis of the evidence available from systematic reviews and meta-analyses using RCTs and cohort studies investigating the effects of MUFA on cardiovascular and diabetic risk factors, several studies indicated an increase of HDL-C following a MUFA-rich diet [20].

\section{Discussion}

To make "Dietary Reference Intakes for Japanese 2015", we studied effects of intake of various dietary fat on serum HDL$\mathrm{C}$ levels, by using meta-analyses of RCTs which have been published up to 2012 [4]. In our previous report, the substitution of FA for carbohydrates is beneficially associated with HDL-C, and MUFA intake may not affect HDL-C [4]. However, the present study showed high-MUFA diet significantly increased HDL-C as compared with high-carbohydrates diets $[15,18]$, and found that several studies indicated an increase of HDL-C following a MUFA-rich diet [20].

We previously reported that TFA is significantly associated with reduction of HDL-C, and that TFA is also adversely related with TC/HDL-C [4]. In the present study, we studied the influences of IP-TFA and R-TFA including CLA on HDL. Interestingly, R-TFA did not influence the changes in the ratios of plasma TC/HDL-C and LDL-C/HDL-C [6], and foods enriched with CLA were associated with a non-significant increase of HDL-C [17]. IP-TFA intake led to a significant increase in LDL-C and a significant decrease in HDL-C [8]. When considering the influence of TFA on serum lipid, it was suggested that IP-TFA and R-TFA have to be considered separately.

Our previous study suggested that n-3 PUFA consumption, especially DHA consumption, may be favorably associated with HDL metabolism [4]. The present study showed that n-3 PUFA did not increase HDL-C in patients with obesity and ESRD $[10,11,21]$; however, n-3 PUFA significantly increased HDL in patients with NAFLD $[13,14]$. This result indicates that the effect of n-3 PUFA on HDL-C varies by the difference of clinical backgrounds of studied patients. Our recent study reported that DHA induces a greater increase of HDL-C as compared with eicosapentaenoic acid (EPA) [28], supporting our previous study.

In this study, we also examined the influence of SFA intake on HDL. Palm oil consumption increased HDL-C compared with vegetable oils low in SFA; however, palm oil increased LDL-C [5]. Comparison of palm oil diets with diets rich in stearic acid, MUFA and PUFA showed significantly higher 
Table 2. Summary of Effects of Various Fatty Acids Consumption on Serum Lipids

\begin{tabular}{llll} 
& HDL-C & LDL-C & TG \\
\hline SFA & $\uparrow$ & $\uparrow$ & \\
TFA & & & \\
Industrially produced-TFA & $\downarrow$ & $\uparrow$ & \\
Ruminant-TFA & $\rightarrow$ & $\rightarrow$ & \\
CLA (ruminant-TFA) & $\rightarrow$ & $\downarrow$ & $\rightarrow$ \\
n-3 PUFA & $\rightarrow$ or $\uparrow$ & $\rightarrow$ or $\downarrow$ & $\downarrow$ \\
MUFA & $\rightarrow$ or $\uparrow$ & $\downarrow$ or $\rightarrow$ & $\rightarrow$ or $\downarrow$ \\
\hline
\end{tabular}

CLA: conjugated linoleic acid; HDL-C: high-density lipoprotein-cholesterol; LDL-C: low-density-cholesterol; MUFA: mono-unsaturated fatty acid; PUFA: poly-unsaturated fatty acid; SFA: saturated fatty acid; TFA: trans fatty acid; TG: triglyceride.

HDL-C and apolipoprotein A-I; however, showed significantly higher LDL-C and apolipoprotein B [7]. Compared with carbohydrates, C12-C16 SFAs raise LDL-C and HDL-C without affecting the TC/HDL-C ratio; other SFAs have neutral effects on serum lipid profile [19]. Replacing 3\% dietary SFA with MUFA or PUFA lowers LDL-C by $2 \%$ and TC/HDL-C ratio [19]. Both elevation of LDL-C and reduction of HDL-C are very crucial determinants for atherogenesis. Meta-analyses of prospective cohort studies reported the relative risks $(95 \% \mathrm{CI})$ of high versus low intakes of SFA to be 1.07 (95\% CI: 0.96 - 1.19) for CHD, which was not statistically significant [29]. Meta-analysis of RCTs reports mean reductions of 14\% $(95 \%$ CI: 4 - 23) in CHD incidence and 6\% (95\% CI: -25 - 4; nonsignificance) in mortality, where SFA was lowered by decreasing and/or modifying dietary fat [30].

The systematic review and meta-analysis which analyzed the associations between intake of SFA and TFA and all-cause mortality, cardiovascular disease (CVD) and associated mortality, CHD and associated mortality, ischemic stroke and type 2 diabetes, was reported [31]. SFA intake was not associated with all-cause mortality (relative risk: $0.99,95 \%$ CI: 0.91 1.09), CVD mortality (0.97, $0.84-1.12)$, total CHD $(1.06,0.95$ - 1.17), ischemic stroke $(1.02,0.90-1.15)$ or type 2 diabetes $(0.95,0.88-1.03)$ [31]. TFA intake was associated with allcause mortality $(1.34,1.16-1.56)$, CHD mortality $(1.28,1.09$ - 1.50) and total CHD (1.21, 1.10 - 1.33), but not ischemic stroke $(1.07,0.88-1.28)$ or type 2 diabetes $(1.10,0.95-1.27)$ [31]. Industrial, but not ruminant, TFAs were associated with CHD mortality $(1.18,1.04-1.33)$ and CHD $(1.42,1.05-1.92)$ [31].

\section{Conclusions}

Summary of effects of various FA consumption on serum lipids was shown in Table 2. Consumption of ruminant-TFA including CLA may not affect HDL-C, and an intake of n-3 PUFA and MUFA was associated with an increase of HDL-C. These FAs may not induce atherosclerosis even by considering the effects of such FA on other serum lipids. The effect of
SFA on atherosclerosis has to be carefully considered by accumulation of the effect of HDL-C/LDL-C ratio on CV events. Judging from effect on CV events and serum lipids, IP-TFA consumption may induce atherosclerosis.

\section{Conflict of Interest}

The authors declare that they have no competing interests.

\section{References}

1. Kitamura A, Iso H, Naito Y, Iida M, Konishi M, Folsom AR, Sato S, et al. High-density lipoprotein cholesterol and premature coronary heart disease in urban Japanese men. Circulation. 1994;89(6):2533-2539.

2. Yokokawa H, Yasumura S, Tanno K, Ohsawa M, Onoda T, Itai K, Sakata K, et al. Serum low-density lipoprotein to high-density lipoprotein ratio as a predictor of future acute myocardial infarction among men in a 2.7-year cohort study of a Japanese northern rural population. J Atheroscler Thromb. 2011;18(2):89-98.

3. Okamura T, Hayakawa T, Kadowaki T, Kita Y, Okayama A, Ueshima H, The NIPPON DATA90 Research Group. The inverse relationship between serum high-density lipoprotein cholesterol level and all-cause mortality in a 9.6-year follow-up study in the Japanese general population. Atherosclerosis. 2006;184(1):143-150.

4. Yanai H, Katsuyama H, Hamasaki H, Abe S, Tada N, Sako A. Effects of dietary fat intake on HDL metabolism. J Clin Med Res. 2015;7(3):145-149.

5. Sun Y, Neelakantan N, Wu Y, Lote-Oke R, Pan A, van Dam RM. Palm oil consumption increases LDL cholesterol compared with vegetable oils low in saturated fat in a meta-analysis of clinical trials. J Nutr. 2015;145(7):15491558.

6. Gayet-Boyer C, Tenenhaus-Aziza F, Prunet C, Marmonier $\mathrm{C}$, Malpuech-Brugere $\mathrm{C}$, Lamarche $\mathrm{B}$, Chardigny $\mathrm{JM}$. Is there a linear relationship between the dose of ruminant trans-fatty acids and cardiovascular risk markers in healthy subjects: results from a systematic review and meta-regression of randomised clinical trials. Br J Nutr. 2014;112(12):1914-1922.

7. Fattore E, Bosetti C, Brighenti F, Agostoni C, Fattore G. Palm oil and blood lipid-related markers of cardiovascular disease: a systematic review and meta-analysis of dietary intervention trials. Am J Clin Nutr. 2014;99(6):13311350 .

8. Aronis KN, Khan SM, Mantzoros CS. Effects of trans fatty acids on glucose homeostasis: a meta-analysis of randomized, placebo-controlled clinical trials. Am J Clin Nutr. 2012;96(5):1093-1099.

9. Hannon BA, Thompson SV, An R, Teran-Garcia M. Clinical outcomes of dietary replacement of saturated fatty acids with unsaturated fat sources in adults with overweight and obesity: a systematic review and meta-analysis of randomized control trials. Ann Nutr Metab. 2017;71(12):107-117. 
10. Zhang YY, Liu W, Zhao TY, Tian HM. Efficacy of Omega-3 Polyunsaturated Fatty Acids Supplementation in Managing Overweight and Obesity: A Meta-Analysis of Randomized Clinical Trials. J Nutr Health Aging. 2017;21(2):187-192.

11. Xu T, Sun Y, Sun W, Yao L, Sun L, Liu L, Ma J, et al. Effect of omega-3 fatty acid supplementation on serum lipids and vascular inflammation in patients with end-stage renal disease: a meta-analysis. Sci Rep. 2016;6:39346.

12. Perna S, Giacosa A, Bonitta G, Bologna C, Isu A, Guido D, Rondanelli M. Effects of hazelnut consumption on blood lipids and body weight: a systematic review and Bayesian meta-analysis. Nutrients. 2016;8(12):747.

13. He XX, Wu XL, Chen RP, Chen C, Liu XG, Wu BJ, Huang ZM. Effectiveness of omega-3 polyunsaturated fatty acids in non-alcoholic fatty liver disease: a metaanalysis of randomized controlled trials. PLoS One. 2016;11(10):e0162368.

14. Lu W, Li S, Li J, Wang J, Zhang R, Zhou Y, Yin Q, et al. Effects of omega-3 fatty acid in nonalcoholic fatty liver disease: a meta-analysis. Gastroenterol Res Pract. 2016;2016:1459790.

15. Qian F, Korat AA, Malik V, Hu FB. Metabolic effects of monounsaturated fatty acid-enriched diets compared with carbohydrate or polyunsaturated fatty acid-enriched diets in patients with type 2 diabetes: a systematic review and meta-analysis of randomized controlled trials. Diabetes Care. 2016;39(8):1448-1457.

16. Khalesi S, Paukste E, Nikbakht E, Khosravi-Boroujeni H. Sesame fractions and lipid profiles: a systematic review and meta-analysis of controlled trials. Br J Nutr. 2016;115(5):764-773.

17. Derakhshande-Rishehri SM, Mansourian M, Kelishadi R, Heidari-Beni M. Association of foods enriched in conjugated linoleic acid (CLA) and CLA supplements with lipid profile in human studies: a systematic review and meta-analysis. Public Health Nutr. 2015;18(11):20412054.

18. Schwingshackl L, Hoffmann G. Comparison of effects of long-term low-fat vs high-fat diets on blood lipid levels in overweight or obese patients: a systematic review and meta-analysis. J Acad Nutr Diet. 2013;113(12):16401661.

19. Sanders TA. Reappraisal of SFA and cardiovascular risk. Proc Nutr Soc. 2013;72(4):390-398.

20. Schwingshackl L, Hoffmann G. Monounsaturated fatty acids and risk of cardiovascular disease: synopsis of the evidence available from systematic reviews and meta- analyses. Nutrients. 2012;4(12):1989-2007.

21. Pei J, Zhao Y, Huang L, Zhang X, Wu Y. The effect of n-3 polyunsaturated fatty acids on plasma lipids and lipoproteins in patients with chronic renal failure - a meta-analysis of randomized controlled trials. J Ren Nutr. 2012;22(6):525-532.

22. Huth PJ, Park KM. Influence of dairy product and milk fat consumption on cardiovascular disease risk: a review of the evidence. Adv Nutr. 2012;3(3):266-285.

23. Mensink RP, Katan MB. Effect of dietary trans fatty acids on high-density and low-density lipoprotein cholesterol levels in healthy subjects. N Engl J Med. 1990;323(7):439-445.

24. Stender S, Astrup A, Dyerberg J. A trans European Union difference in the decline in trans fatty acids in popular foods: a market basket investigation. BMJ Open. 2012;2(5).

25. Rice BH, Kraft J, Destaillats F, Bauman DE, Lock AL. Ruminant-produced trans-fatty acids raise plasma HDL particle concentrations in intact and ovariectomized female Hartley guinea pigs. J Nutr. 2012;142(9):16791683.

26. Lock AL, Parodi PW, Bauman DE. The biology of transfatty acids: implications for human health and the dairy industry. Aust J Dairy Technol. 2005;60:134-142.

27. Gebauer SK, Chardigny JM, Jakobsen MU, Lamarche B, Lock AL, Proctor SD, Baer DJ. Effects of ruminant trans fatty acids on cardiovascular disease and cancer: a comprehensive review of epidemiological, clinical, and mechanistic studies. Adv Nutr. 2011;2(4):332-354.

28. Yanai H, Masui Y, Katsuyama H, Adachi H, Kawaguchi A, Hakoshima M, Waragai Y, et al. An improvement of cardiovascular risk factors by omega-3 polyunsaturated fatty acids. J Clin Med Res. 2018;10(4):281-289.

29. Siri-Tarino PW, Sun Q, Hu FB, Krauss RM. Meta-analysis of prospective cohort studies evaluating the association of saturated fat with cardiovascular disease. Am J Clin Nutr. 2010;91(3):535-546.

30. Hooper L, Summerbell CD, Thompson R, Sills D, Roberts FG, Moore HJ, Davey Smith G. Reduced or modified dietary fat for preventing cardiovascular disease. Cochrane Database Syst Rev. 2012;5:CD002137.

31. de Souza RJ, Mente A, Maroleanu A, Cozma AI, Ha V, Kishibe T, Uleryk E, et al. Intake of saturated and trans unsaturated fatty acids and risk of all cause mortality, cardiovascular disease, and type 2 diabetes: systematic review and meta-analysis of observational studies. BMJ. 2015;351:h3978. 